\title{
The Fifth Spectrum of Praseodymium
}

\author{
Victor Kaufman and Jack Sugar \\ Institute for Basic Standards, National Bureau of Standards, Washington, D.C. 20234
}

(June 5, 1967)

\begin{abstract}
Twelve spectral lines of quadruply-ionized praseodymium in the region 840 to $2250 \AA$ are reported. Five terms $\left(4 f^{2} \mathrm{~F}, 5 d^{2} \mathrm{D}, 6 s^{2} \mathrm{~S}, 6 p^{2} \mathrm{P}\right.$, and $\left.7 s^{2} \mathrm{~S}\right)$ and the hyperfine splitting of the $6 s^{2} \mathrm{~S}$ term are given. A calculation of the principal ionization energy $\left(\operatorname{Pr}^{4+} \rightarrow \mathrm{Pr}^{5+}\right)$ leads to a value of $57.44 \mathrm{eV}$ with an estimated uncertainty of $\pm 0.05 \mathrm{eV}$.
\end{abstract}

Key Words: Atomic energy levels, isoelectronic sequence, praseodymiun, rare earth, spectrum, sliding spark discharge lamp.

\section{Introduction}

The one-electron Cs I isoelectronic sequence has been observed through Ce IV. The present work extends this to PrV. Several orbital properties such as quantum defect, doublet splitting, and term energy intervals were found to vary in a regular way through the Cs I sequence. These regularities have been used here to verify the analysis of $\operatorname{Pr} \mathrm{V}$.

This is the first example of the structure of the fifth spectrum of a rare earth. The regularities in the level structure of the $4 f$ period of atoms in lower stages of ionization indicate that $\operatorname{Pr} \mathrm{V}$ may serve as a guide to the interpretation of the more complex fifth spectra of the rare earths.

\section{Experimental Procedure}

The spectrum plates obtained in the investigation of Pr IV [1] ${ }^{1}$ were found to contain several very strong and fairly isolated lines in a 500-A spark exposure which showed no trace in the 50-A exposure. These could only be interpreted as high excitation Pr IV lines, which is unlikely because of their unusual strength, or $\operatorname{Pr} \mathrm{v}$ lines. Unfortunately, the slidingspark light source [2] used in this experiment does not suppress $\operatorname{Pr}$ IV relative to $\operatorname{Pr} \mathrm{V}$. Therefore, the assignment of these lines to $\operatorname{Pr} \mathrm{V}$ could not be based exclusively on excitation separation.

Exposures were made in the first order of a $35-\mathrm{ft}$ Eagle vacuum spectrograph at a reciprocal dispersion of $0.78 \AA / \mathrm{mm}$. For further corroboration of the lines of the $6 s-6 p$ and $6 p-7 s$ transitions and for a better intensity relationship between these lines, exposures were also made in the first order of a 2.2-meter Eagle vacuum spectrograph at a reciprocal dispersion of $2.75 \AA / \mathrm{mm}$. Calculated and measured wavelengths of $\mathrm{C}, \mathrm{N}, \mathrm{O}, \mathrm{Si}, \mathrm{Ge}$, and $\mathrm{Cu}$ in various stages of ionization

${ }^{1}$ Figures in brackets indicate the literature references at the end of this paper. were used as standards of calibration [3-7]. The estimated wavelength uncertainty for all lines, except those at $1958 \AA$, is $\pm 0.005 \AA$. Those at $1958 \AA$ have an estimated uncertainty of $\pm 0.003 \AA$.

\section{Wavelengths and Energy Levels}

A list of the classified lines of Pr $\mathbf{v}$, including visual estimates of their relative intensities, wavenumbers, and classifications is given in table 1 . The list of energy levels of Pr V derived therefrom is given in table 2 . It includes the level designation, the observed position with estimated uncertainty, the fine structure splitting where appropriate, and the hyperfine structure splitting for the $6 s^{2} \mathrm{~S}$ term. The hyperfine splitting of the $7 s$ level is calculated to be about $1 . \mathrm{cm}^{-1}$. Because the width of the $6 p-7 s$ lines is about $2 . \mathrm{cm}^{-1}$, this splitting could not be observed.

The interpretation of the observed spectral lines was begun with an accurate estimate of $\zeta_{4 f}$ obtained from the $4 f 6 s$ configuration of $\mathrm{Pr}^{3+}$. This value allowed an unambiguous interpretation of the intense $4 f-5 d$ lines. Using this $5 d$ doublet splitting, we were

TABLE 1. Observed spectral lines of $\operatorname{Pr} \mathrm{V}$

\begin{tabular}{|c|c|c|c|}
\hline $\begin{array}{c}\text { Wavelength } \\
(\AA) \\
\end{array}$ & Intensity & $\underset{\left(\mathrm{cm}^{-1}\right)}{\text { Wavenumber }}$ & Classification \\
\hline 843.783 & 200 & 118513.9 & $14 f^{2} \mathrm{~F}_{5 / 2}^{\circ}-5 d^{2} \mathrm{D}_{5 / 2}$ \\
\hline 865.902 & 7000 & 115486.5 & $4 f^{2} \mathrm{~F}_{7 / 2}^{\circ}-5 d^{2} \mathrm{D}_{5 / 2}$ \\
\hline 869.170 & 5000 & 115052.3 & $4 f^{2} \mathrm{~F}_{5 / 2}^{\circ}-5 d^{2} \mathrm{D}_{3 / 2}$ \\
\hline 869.662 & 80 & 114987.2 & $5 d^{2} \mathrm{D}_{3 / 2}-6 p{ }^{2} \mathrm{P}_{3 / 2}^{\circ}$ \\
\hline 896.654 & 1000 & 111525.7 & $5 d^{2} \mathrm{D}_{5 / 2}-6 p^{2} \mathrm{P}_{3 / 2}^{0}$ \\
\hline 922.290 & 750 & 108425.8 & $5 d^{2} \mathrm{D}_{3 / 2}-6 p{ }^{2} \mathrm{P}_{1 / 2}^{\circ}$ \\
\hline 1234.070 & 250 & 81032.7 & $6 p{ }^{2} \mathrm{P}_{1 / 2}^{\circ}-7 s^{2} \mathrm{~S}_{1 / 2}$ \\
\hline 1342.775 & 250 & 74472.6 & $6 p^{2} \mathrm{P}_{3 / 2}^{\circ}-7 s^{2} \mathrm{~S}_{1 / 2}$ \\
\hline 1958.088 & 200 & 51070.23 & $6 s^{2} \mathrm{~S}_{1 / 2}(\mathrm{~F}=2)-6 p^{2} \mathrm{P}_{3 / 2}^{\circ}$ \\
\hline 1958.201 & 400 & 51067.28 & $6 s^{2} \mathrm{~S}_{1 / 2}(\mathrm{~F}=3)-6 p{ }^{2} \mathrm{P}_{3 / 2}^{\mathrm{o}}$ \\
\hline 2246.759 & 300 & 44508.56 & $6 s^{2} \mathrm{~S}_{1 / 2}(\mathrm{~F}=2)-6 p^{2} \mathrm{P}_{1 / 2}^{\circ}$ \\
\hline 2246.900 & 300 & 44505.76 & $6 s^{2} \mathrm{~S}_{1 / 2}(\mathrm{~F}=3)-6 p^{2} \mathrm{P}_{1 / 2}^{\circ}$ \\
\hline
\end{tabular}




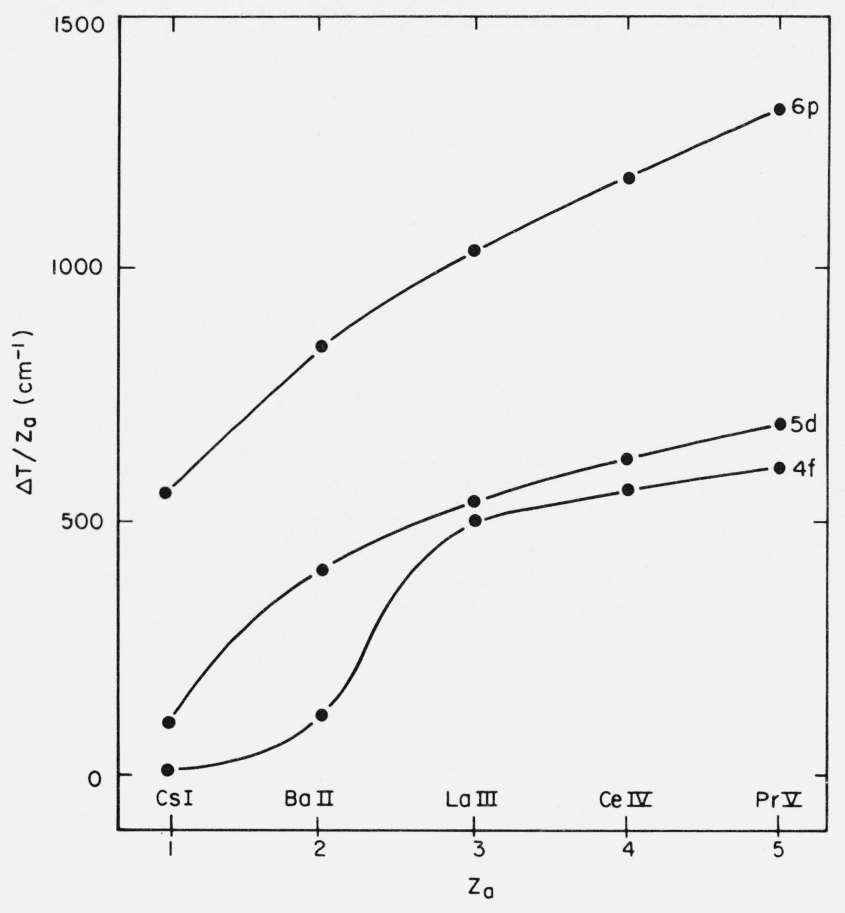

FIgURE 1. Variation of $\frac{\Delta \mathrm{T}}{\mathrm{Z}_{\mathrm{a}}}$ along the Cs I isoelectronic sequence where $\Delta \mathrm{T}$ equals spin-orbit splittings of the $4 \mathrm{f}^{2} \mathrm{~F}, 5 \mathrm{~d}{ }^{2} \mathrm{D}$, and $6 \mathrm{p}{ }^{2} \mathrm{P}$ terms.

Note the anomalous position of the Ba II $4 f^{2} \mathrm{Fpoint}$. References for the plotted values are Cs I; H. Kleiman, J. Opt. Soc. Am. 52, 441 (1962), Ba II; E. Rasmussen, Zeit. f. Phys 83, 404 (1933), La III; see ref. [10]. Ce IV; see ref. [8].

able to interpret the almost-equally prominent $5 d-6 p$ lines. In figure 1 , the ratios of the spin-orbit splitting, $\Delta T$ to $Z_{a}$ are plotted against $Z_{a}$ in the Cs I isoelectronic sequence. The spin-orbit splittings are those of the $4 f^{2} \mathrm{~F}, 5 d^{2} \mathrm{D}$, and $6 p^{2} \mathrm{P}$ terms, and $Z_{a}$ is the positive charge on the ion remaining after the removal of the outer electron. The values for Pr V provide smooth extensions of the curves drawn through the previously known points.

The combinations of the $6 p^{2} \mathrm{P}$ term with the $6 s$ and

TABLE 2. Energy levels of $\operatorname{Pr} \mathrm{V}$

\begin{tabular}{l|r|r|r|r}
\hline \hline Designation & \multicolumn{1}{|c|}{$J$} & $\begin{array}{c}\text { Energy } \\
\left(\mathrm{cm}^{-1}\right)\end{array}$ & $\begin{array}{c}\text { Uncertainty } \\
\left(\mathrm{cm}^{-1}\right)\end{array}$ & $\begin{array}{c}\text { Splitting } \\
\left(\mathrm{cm}^{-1}\right)\end{array}$ \\
\hline $4 f^{2} \mathrm{~F}^{\circ}$ & $2^{1 / 2}$ & 0.0 & & \\
& $3^{1 / 2}$ & 3027.4 & \pm 1.0 & 3027.4 \\
$5 d^{2} \mathrm{D}$ & $1^{1 / 2}$ & 115052.3 & 1.0 & \\
& $2^{1 / 2}$ & 118513.8 & 1.0 & 3461.5 \\
$6 s^{2} \mathrm{~S}(\mathrm{~F}=2)$ & $1 / 2$ & 178969.4 & 1.5 & \\
$(\mathrm{~F}=3)$ & $1 / 2$ & 178972.3 & 1.5 & 2.95 \\
$6 p{ }^{2} \mathrm{P}^{\circ}$ & $1 / 2$ & 223478.1 & 1.5 & 6561.4 \\
& $1 s^{2} \mathrm{~S}$ & 230039.5 & 1.5 & \\
& $1 / 2$ & 304511.5 & 2.0 & \\
\hline
\end{tabular}

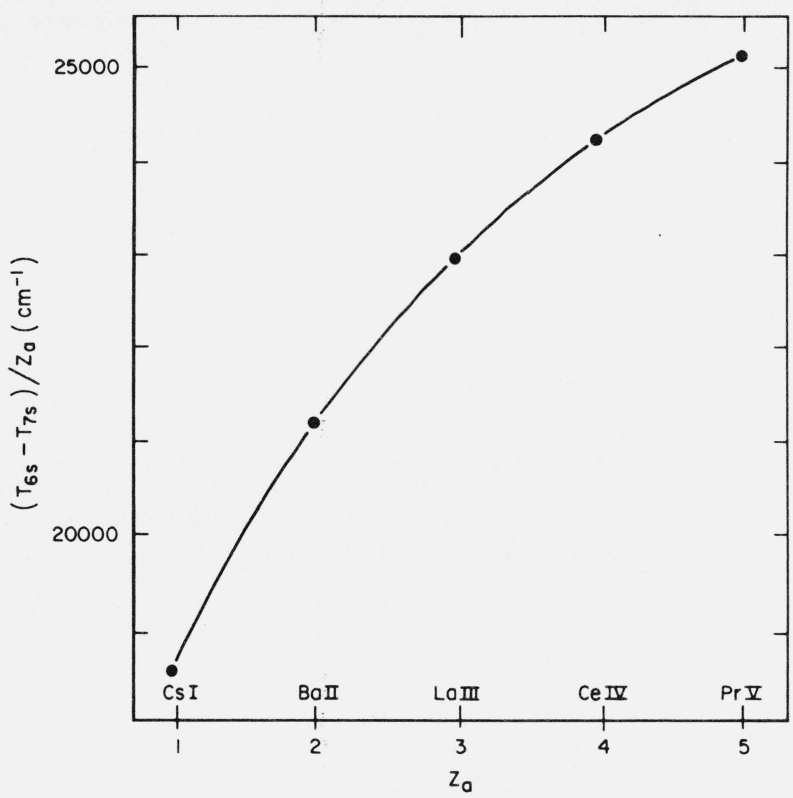

FigURE 2. Variation of the ratio of separation of the $6 \mathrm{~s}{ }^{2} \mathrm{~S}$ and $7 \mathrm{~s}{ }^{*} \mathrm{~S}$ terms to $\mathrm{Z}_{\mathrm{a}}$ along the isoelectronic sequence.

For references for the plotted values see the caption of figure 1 .

$7 s^{2} \mathrm{~S}$ levels were not so easily distinguished because of the comparable strength of numerous Pr IV lines in the same spectral region. Evidence for our interpretation of these lines in terms of the behavior of the $6 s-7 s$ energy interval is presented in figure 2 where the observed value for Pr $\mathrm{v}$ follows the smooth variation of this interval in the Cs I isoelectronic sequence.

In figure 3 we present the sequential behavior of the quantum defect, $n-n_{a}$, for each of the observed terms. These points are interrelated since the quantum defects depend on the positions of the terms relative to the ionization limit which in turn is determined by the positions of the $n s^{2} \mathrm{~S}$ terms. Therefore the agreement of the Pr $\mathrm{V}$ experimental values with the isoelectronic sequence is a strong confirmation of the analysis of this spectrum.

Because of the noticeable departure of the Ba II $4 f^{2} \mathrm{~F}$ points in figures 1 ând 3 , we have examined the $n f$ series of $\mathrm{Ba}$ II in detail and found that the behavior of $\left(n-n_{a}\right)$ versus $1 / n_{a}^{2}$ is quite different from that of the same series in Cs I and La III. It is our impression that this part of the Ba II analysis bears reexamination.

The value of the spin-orbit parameter, $\zeta_{4 f}$, derived from the $4 f^{2} F$ term of $\operatorname{Pr} \mathrm{V}$ equals $865.0 \pm 0.3 \mathrm{~cm}^{-1}$. From the $4 f 6 s$ configuration of Priv, one finds [1] $\zeta_{4 f}=860.8 \pm 0.2 \mathrm{~cm}^{-1}$. The difference is due to a slight shielding of the $4 f$ electron by the $6 s$ electron in Pr IV. The same order of magnitude of this effect can be found in the comparison of the same configurations of Ce IV and Ce III [8, 9]. However, the value of $\zeta_{4 f}$ from La III [10] is slightly smaller than that obtained from 4f6s of La II [11]. This is probably due to a small perturbation in La II where configurations of the same parity are very close. 


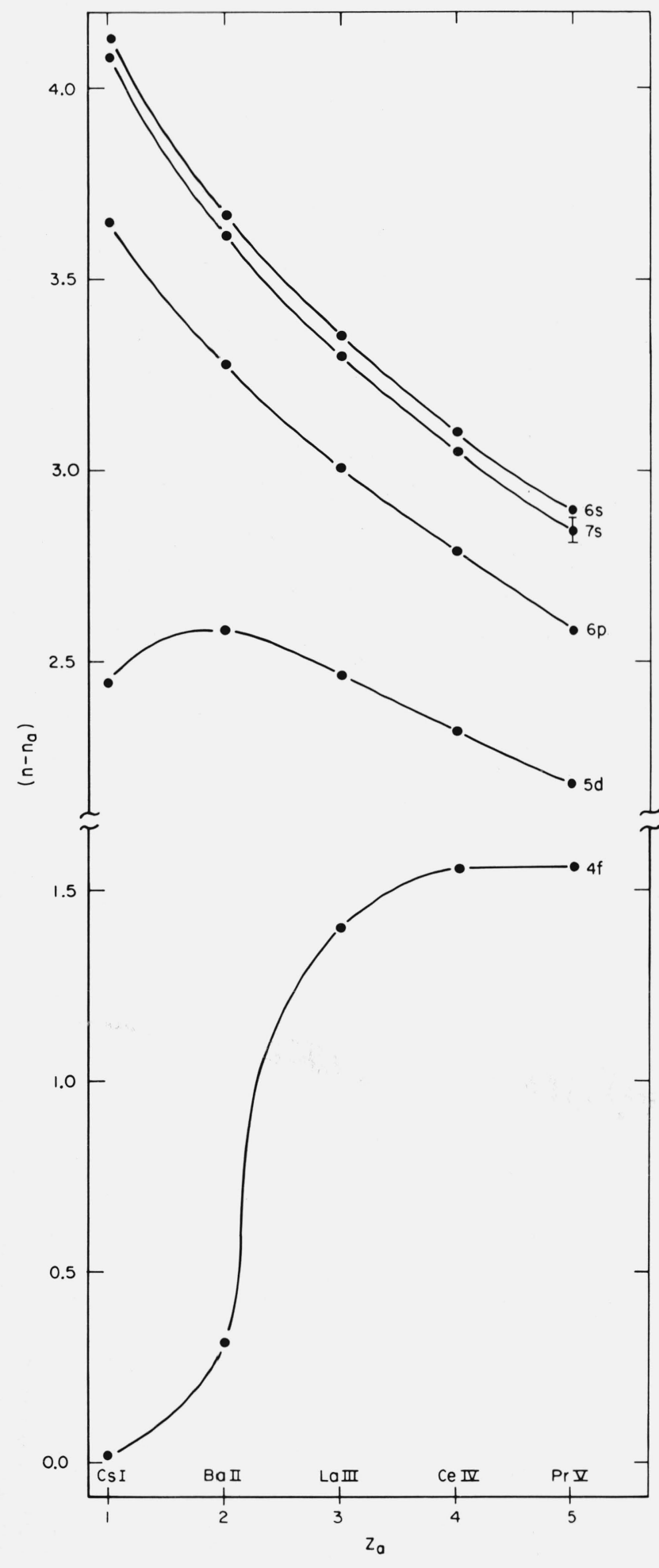

FIGURE 3. Variation of quantum defect of the center of gravity of the $4 \mathrm{f}, 5 \mathrm{~d}, 6 \mathrm{p}, 6 \mathrm{~s}$, and $7 \mathrm{~s}$ terms along the $\mathrm{Cs} \mathrm{I}$ isoelectronic sequence. Note the anomalous position of the Ba II $4 f$ point. The error bar on the $7 s{ }^{2} \mathrm{~S} \operatorname{Pr} \mathrm{v}$ point indicates the variation in the quantum defect for a change in the ionization energy of indicates the variation in the quantum defect for a change in the ionization
$\pm 400 \mathrm{~cm}^{-1}$. For references for the plotted values see the caption of figure 1 .

\section{Ionization Potential}

In table 2 of the recent paper of Sugar and Reader [12] values of $\Delta n_{a}=n_{a}(7 s)-n_{a}(6 s)$ derived from several $4 f^{N} n s$ series in the region of the rare earths are given which show that a value of $\Delta n_{a}=1.050 \pm 0.005 \mathrm{~cm}^{-1}$ can be used with confidence to compute the ionization energies of rare earths. The values listed are for singly, doubly and triply ionized species. With this value and the experimentally determined value of $\Delta T=E(7 s)-E(6 s)=125540.4 \mathrm{~cm}^{-1}$, the equation

$$
\Delta T=\frac{Z_{a}^{2} R}{\left[n_{a}(6 s)\right]^{2}}-\frac{Z_{a}^{2} R}{\left[n_{a}(6 s)+\Delta n_{a}\right]^{2}},
$$

where $Z_{a}=5$, was solved for $n_{a}(6 s)$. The corresponding value of $T(6 s)=Z_{a}^{2} R /\left[n_{a}(6 s)\right]^{2}$, when added to the level value of $6 s^{2} \mathrm{~S}$ gives the ionization potential as 463350 $\mathrm{cm}^{-1}$. On the basis of the uncertainty in the value of $\Delta n_{a}$, the value of the ionization potential is given as $463400 \pm 400 \mathrm{~cm}^{-1}(57.44 \pm 0.05 \mathrm{eV})$.

In figure 3 , the error bar on the $7 s^{2} \mathrm{~S}, \operatorname{Pr} \mathrm{v}$ point shows the extent of the possible change in the position of that point for a change in the ionization energy of the stated uncertainty. This gives us confidence that the value of $\Delta n_{a}$ used is valid for highly ionized rare earths.

\section{Hyperfine Splitting of the $6 \mathrm{~s}$ Term}

The hyperfine splitting of the $6 s^{2} \mathrm{~S}$ term of $2.95 \pm$ $0.05 \mathrm{~cm}^{-1}$ leads to a value for the splitting factor of the $6 s$ electron $\left(a_{6 s}\right)$ of $0.98_{3} \pm 0.01_{7} \mathrm{~cm}^{-1}$. It is of interest to compare this value with $a_{6 s}=0.639 \pm 0.007 \mathrm{~cm}^{-1}$ for Pr III given by Reader and Sugar [13]. Relating these splittings according to the Goudsmit-Fermi-Segré formula, we get

$$
\left[\frac{a_{6 s}}{Z_{a}^{2}} \cdot \frac{n_{a}^{3}}{d n_{a} / d n}\right]_{\mathrm{Pr} \mathrm{v}}=\left[\frac{a_{6 s}}{Z_{a}^{2}} \cdot \frac{n_{a}^{3}}{d n_{a} / d n}\right]_{\mathrm{Pr} \mathrm{II}}
$$

Insertion of the experimental values in the above equation gives $a_{6 s}(\operatorname{Pr} \mathrm{v})=1.03_{8} \pm 0.03_{2} \mathrm{~cm}^{-1}$, which leads to an expected splitting of $3.11 \pm 0.10 \mathrm{~cm}^{-1}$. This compares quite well with the experimentally determined value listed above.

\section{References}

[1] J. Sugar, J. Opt. Soc. Am. 55, 1058 (1965).

[2] J. Sugar, J. Opt. Soc. Am. 53, 831 (1963).

[3] V. Kaufman and K. L. Andrew, J. Opt. Soc. Am. 52, 1223 (1962).

[4] V. Kaufman, L. J. Radziemski, Jr., and K. L. Andrew, J. Opt. Soc. Am. 56, 911 (1966).

[5] V. Kaufman and J. F. Ward, J. Opt. Soc. Am. 56, 1591 (1966).

[6] V. Kaufman and J. F. Ward, Appl. Opt. 6, 43 (1967).

[7] B. Edlén, Rep. Prog. Phys. 26, 181 (1963).

[8] R. J. Lang, Can. J. Res. 14A, 127 (1936).

[9] N. Spector, J. Opt. Soc. Am. 55, 492 (1965).

[10] J. Sugar and V. Kaufman, J. Opt. Soc. Am. 55, 1283 (1965).

[11] H. N. Russell and W. F. Meggers, BS J. Res. 9, 625 (1932) RP497.

[12] J. Sugar and J. Reader, J. Opt. Soc. Am. 55, 1286 (1965).

[13] J. Reader and J. Sugar, Phys. Rev. 137, B784 (1965).

(Paper 71A6-474) 